\title{
Empty Coruña? Un dispositivo académico transdisciplinar sobre la problemática urbana. Reflexiones desde los estudios de Economía y Administración de Empresas
}

Bruna, Fernando'; DeLlano-Paz, Fernando²; Longarela-Ares, Ángeles³; Calvo-Silvosa, Anxo²

${ }^{1}$ Grupo de investigación Jean Monnet de Competencia y Desarrollo $(C+D)$ y Grupo de Innovación Docente en Pensamiento Económico Crítico, Departamento de Economía,

Facultad de Economía y Empresa. Universidade da Coruña.

${ }^{2}$ Grupo en Regulación, Economía y Finanzas (GREFIN),Departamento de Empresa,

Facultad de Economía y Empresa. Universidade da Coruña.

${ }^{3}$ Grupo en Regulación, Economía y Finanzas (GREFIN) y Grupo de Innovación docente en Economía y Empresa (GRIDEM), Departamento de Empresa,

Facultad de Economía y Empresa. Universidade da Coruña.

\section{RESUMEN}

El curso de verano Empty Coruña nace como resultado de la labor desarrollada por el equipo de trabajo con el mismo nombre formado por un grupo de docentes de distintas facultades de la Universidade da Coruña. A través de esta propuesta se trató de construir un corpus teórico inédito, a la vez que una hoja de ruta para trabajo futuro. La universidad se acercó a la ciudad para tratar de entender y cuestionar el modelo económico que ha llevado a una situación de "gente sin casas, y casas sin gente". Conceptualmente, la mayor innovación del curso ha sido su carácter transdisciplinar, para analizar una problemática local con perspectiva global. Metodológicamente, destaca que el aula se trasladó a espacios urbanos al aire libre, como actores pasivos del análisis, así como el enfoque activo y participativo de enseñanza-aprendizaje. Esta experiencia proporciona herramientas para repensar la docencia tradicional en el ámbito de la Facultad de Economía y Empresa. 
PALABRAS CLAVE: Transdisciplinariedad, urbanismo, economía, finanzas, aula invertida.

\section{CITA RECOMENDADA:}

Bruna, F,; DeLlano-Paz, F.; Longarela-Ares, A,; Calvo-Silvosa, A. (2019):Empty Coruña? Un dispositivo académico transdisciplinar sobre la problemática urbana. Reflexiones desde los estudios de Economía y Administración de Empresas. En De la Torre Fernández, E. (ed.) (2019). Contextos universitarios transformadores: construíndo espazos de aprendizaxe. III Xornadas de Innovación Docente. Cufie. Universidade da Coruña. A Coruña (pág. 319-332).

DOl capítulo: https://doi.org/10.17979/spudc.9788497497121.319

DOl libro: https://doi.org/10.17979/spudc.9788497497121

\section{ABSTRACT}

The summer course Empty Coruña appears as the results from the work of a team with the same name formed by professors from several faculties at the Universidade da Coruña. The project tried to build an unprecedented analytical corpus and a roadmap for future work. The University gets closer to the city to try to understand and challenge the economic model that led to a situation of "houses without people and people without houses". Conceptually, the main innovation of the course was its transdisciplinary approach, to analyse a local problem with global perspective. Methodologically, it is noteworthy that the classroom travelled to urban spaces outdoors, considered as passive actors of the analysis, as well as the active and participatory teaching-learning approach. This experience provides tools to rethink the traditional way of teaching within the Faculty of Economics and Business.

KEY WORDS: Transdisciplinarity, urbanism, economy, finance, flipped classroom. 


\section{INTRODUCCIÓN}

La reciente crisis económica ha dejado un paisaje de solares, edificios incompletos, abandonados 0 vacantes, en diversas formas. En el área urbana de A Coruña, alrededor de 2.000 elementos arquitectónicos de todo tipo y un $20 \%$ de las viviendas han sido catalogadas por el equipo Empty Coruña en alguno de los estados críticos referidos. La herida de la crisis se manifiesta también en el cuerpo social, con gente que ha perdido sus casas, sus puestos de trabajo y también servicios públicos. El resultado de todo ello se resume en un eslogan que ha sido muy usado por los movimientos sociales durante este periodo: "gente sin casas, y casas sin gente".

La investigación y catalogación citada ha servido de base para diseñar un curso de verano', impartido en A Coruña del 29 de agosto al 8 de septiembre de 2018 (ver llustración 1), desde el que construir un corpus teórico inédito, a la vez que una hoja de ruta para trabajo futuroii. La iniciativa parte de un grupo de docentes de Arquitectura, Arquitectura Técnica, Sociología, Derecho, Economía y Empresa. Colaboran además el Grupo de Estudios Territoriales y el laboratorio CARTOLAB de la Escuela de Caminos, todos ellos de la Universidade da Coruña (UDC), apoyándose en colaboradores externos como Proxecto Cárcere, Fundación Arquia, el Ayuntamiento de Miño y en la propia Universidad, con la coorganización del Ayuntamiento de A Coruña. 


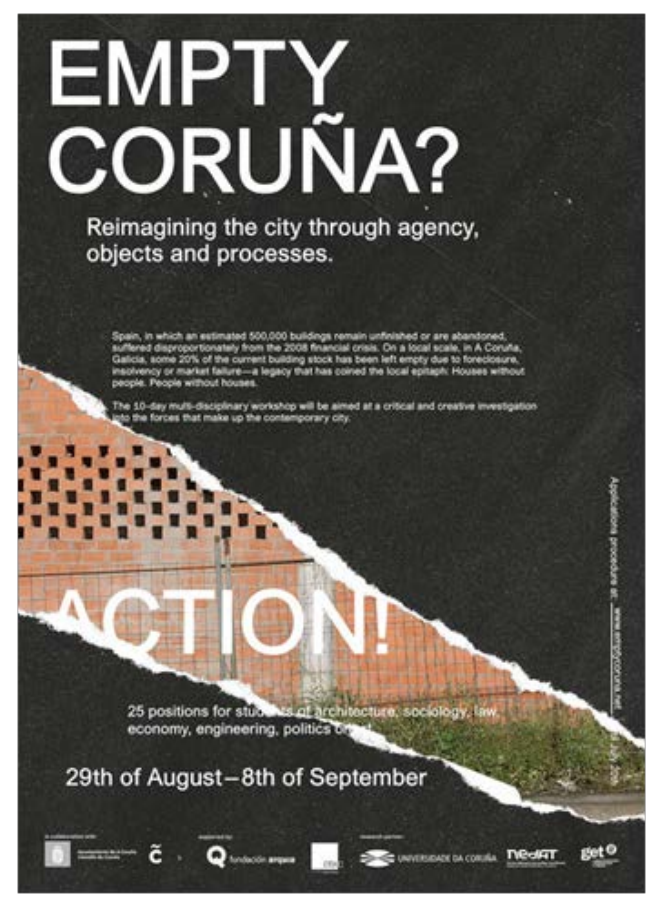

Ilustración 1 - Cartel del curso de verano

Metodológicamente el curso de verano resultó novedoso en, al menos, cinco aspectos:

1) el esquema organizativo gira en torno a tres dimensiones, agentes, objetos y procesos, y no a disciplinas (economía, derecho, arquitectura, etc.), lo que conduce a la creación de un "espacio nuevo", transdisciplinar, donde las relaciones de ideas fluyen más allá de las barreras diferenciadoras de las áreas de conocimiento;

2) el equipo de personas implicado representa la complejidad del análisis a realizar con el curso (son profesores de cuatro facultades distintas, empresarios, miembros de movimientos sociales y otros agentes sociales;

3) el estudiantado del curso presenta también una pluralidad de intereses y de procedencias académicas;

4) los espacios físicos en los que se imparten los talleres son seleccionados como actores pasivos del propio curso, incluyendo rutas guiadas y casi una decena de localizaciones; 
5) la orientación del curso es fundamentalmente práctica y participativa: investigación por grupos de casos reales, juegos de rol, exposiciones, aula invertida, construcción de maquetas, etc.

El presente trabajo relata la participación de cuatro docentes de la Facultad de Economía y Empresa de la UDC en ese curso de verano, experiencia a partir de la cual se realizan algunas reflexiones sobre los estudios de administración y dirección de empresas (ADE) y de economía. El trabajo se presenta con la siguiente estructura: primero se describe la experiencia (punto 2), después se proponen los resultados obtenidos (3) y, finalmente, se extraen las conclusiones (4).

\section{DESCRIPCIÓN DE LA EXPERIENCIA}

Bajo la coordinación de Plácido Lizancosiii, profesor de la Escuela Técnica Superior de Arquitectura, se fue configurando un equipo compuesto por investigadores de distintas facultades. Este equipo tuvo varias reuniones a partir del mes de abril de 2018, con el fin de realizar tormentas de ideas y diseñar el curso de verano. Simultáneamente, varias personas se dedicaron a la catalogación de los elementos urbanos calificados como "vacíos", bajo una tipología de siete casos posibles. Posteriormente, se celebraron varias reuniones más en la Facultad de Economía y Empresa para terminar de configurar el equipo participante por parte de miembros de esta Facultad. A partir de ahí se tuvo una reunión adicional para coordinar los temas a tratar con personas ajenas a la Facultad que fuesen a intervenir en sesiones temáticas relacionadas con el mundo de la empresa. En concreto, estas personas fueron Antón Arias Díaz-Eimil, Roberto Castro Tomé y Marcelino Fernández Mallo, todos ellos expertos vinculados al ámbito empresarial y al sector inmobiliario de A Coruña. Hubo también un intercambio posterior de correos electrónicos con notas y documentación de utilidad.

Los autores de esta comunicación asistieron y participaron en distintas sesiones del curso de verán, pero sus intervenciones se centraron fundamentalmente en cuatro momentos. 
- Previo a la realización del curso, se preparó una documentación para los participantes, con el fin de que dispusieran de un dossier de investigación. Uno de los autores de la presente comunicación, Fernando Bruna, elaboró el documento "Coruña vacía, ¿de qué? ?v, en el que se reflexiona sobre algunas dimensiones relevantes para el estudio de la problemática urbana posterior a la reciente crisis económica.

- A primera hora del tercer día del curso tuvo lugar una sesión 7x7 en la puerta de un edificio actualmente vacío, junto a la Escuela Técnica Superior de Arquitectura de A Coruña, como ilustración de que el fenómeno Empty Coruña afecta también a la propia Universidad. Bajo esta metodología, se presentan 7 ponencias durante 7 minutos antes de abrir un debate general entre los ponentes y el público. Uno de los autores de la presente comunicación, Fernando Bruna, presentó la ponencia "Aspectos macroeconómicos de la crisis inmobiliaria". El reto de esta ponencia suponía en explicar los principales conceptos relacionados con una burbuja inmobiliaria a un alumnado del curso de verano con escasa formación económica previa.

- La tercera intervención tuvo lugar en una plaza pública de la ciudad de A Coruña, una clase in situ focalizada en agentes sociales relacionados con la actividad empresarial, y en la que participaron Antón Arias, Roberto Castro, Anxo Calvo y Marcelino Fernández. Asimismo, en esta sesión Anxo Calvo disertó, basándose en la presentación de un póster (ver llustración 2), sobre "Los fallos de Mercado: mercado vs intervención pública en el sector de la vivienda".

- La cuarta intervención de los autores de la presente comunicación fue en el aula práctica posterior a esta última sesión, en el Centro Cívico Cidade Vella de A Coruña, y consistió en acompañar a los distintos equipos de estudiantes del curso mientras progresaban en la investigación de sus casos de estudio. El foco temático de esa sesión de taller consistía en poner de relieve cuáles eran los agentes involucrados en su caso concreto, y cuál era su papel. Así cada grupo tenía asignado el análisis de un elemento urbanístico concreto (edificio 0 solar) en desuso. El problema se analizaba desde todos los puntos de vista 
posibles en relación con el "porqué" social, económico, administrativo, arquitectónico... que motivó que ese elemento urbanístico acabase en ese estado. A partir del análisis de las posibles causas que condujeron a esa situación, los estudiantes planteaban posibles soluciones.

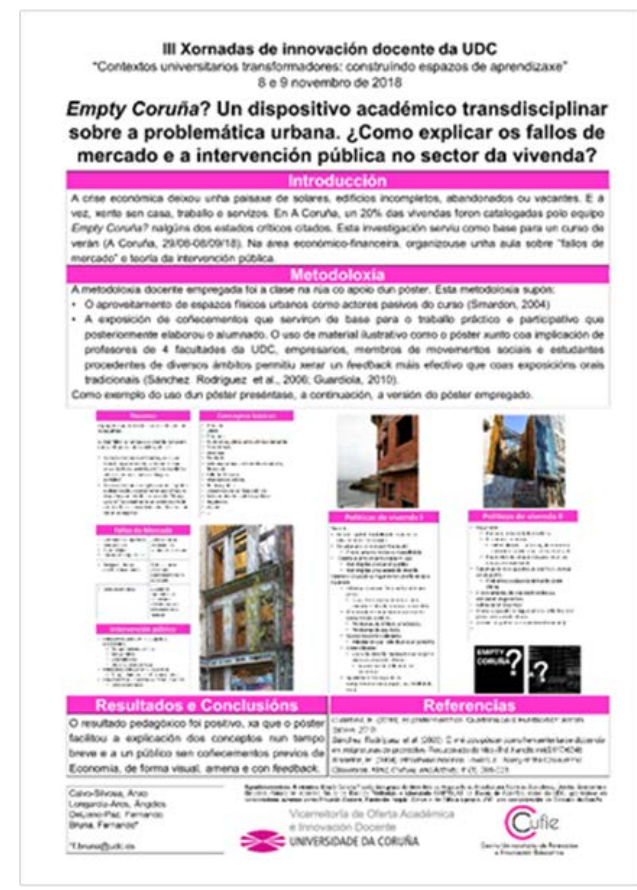

Ilustración 2- Póster presentado por Anxo Calvo en el curso de verano (formato adaptado a las jornadas de innovación docente de la UDC)

\section{RESULTADOS}

Los resultados se pueden organizar atendiendo al momento en que se produjeron. Así se puede distinguir en el tiempo dos fases: la preparatoria relativa al trabajo en equipo de los agentes implicados en el mismo, y la propia de desarrollo del curso de verano.

En relación con los resultados de la fase preparatoria se deben resaltar tres elementos: (1) el intercambio de ideas entre los expertos (docentes y no docentes) en economía y empresa en el contexto de respuesta social al problema planteado desde Empty Coruña; (2) el 
enriquecimiento de la visión estos agentes a través de la puesta en común con otros expertos y agentes sociales relacionados con ámbitos de actuación muy distinta (legal, política, social...); y (3) el establecimiento de una red de colaboración que permita desarrollar ideas surgidas durante este proceso y en el curso de verano (dirección de trabajos de fin de grado y master, proyectos de investigación, etc.). En definitiva, las reuniones preparatorias y el desarrollo del propio curso favorecieron un intercambio de ideas entre profesionales e investigadores de campos diferenciados, lo cual enriqueció enormemente la visión de los autores de la presente comunicación sobre la problemática urbana y sus dimensiones legales, económicas, sociales y políticas. Asimismo, este proceso permitió constatar la dificultad de centrar el análisis económico que requería el curso de verano atendiendo a los estándares docentes empleados en los estudios de economía y empresa.

La fase de desarrollo del curso de verano se estructuró en dos partes, proponiéndose así una metodología docente mixta: seminario de transmisión de conocimientos básicos y taller. Con esta propuesta estructural se persiguieron dos objetivos: que el alumnado aprendiera nuevos conocimientos sobre economía y empresa a través del seminario, y que pusiera en práctica en el taller los contenidos del seminario, contando con el profesorado como guía personalizada (Nimo y Durán, 2017). Esta propuesta metodológica mixta para la parte de análisis económico-financiero del problema objeto del curso Empty Coruña ayudó a: presentar resultados concretos y centrados en el objeto de estudio; generar debate y conseguir la participación del alumnado; fomentar el aprendizaje colaborativo; facilitar la comprensión y vivencia de la experiencia formativa; y mejorar la efectividad docente y la satisfacción con el aprovechamiento del curso.

Como se ha indicado antes, los estudiantes del curso tenían escasa formación previa en temas económicos. Esto, que en principio suponía un obstáculo, resultó tener un papel decisivo y, en ciertos sentidos, positivo:

- En los momentos formativos forzó a centrarse en unos pocos conceptos, desechando otra información relevante. 
- Sin embargo, eso obligó a pensar y construir un relato esencial, que transmitiera una narrativa clara sobre agentes intervinientes, causas y consecuencias. El momento posterior de asesoramiento por grupos durante el taller práctico permitió reforzar este relato.

- Adicionalmente, el esfuerzo por homogeneizar el vocabulario y ofrecer una narrativa centrada en los aspectos básicos fomentó la implicación de los estudiantes, que se percibían en posesión de las herramientas claves para comprender, investigar e incidir en la problemática de estudio.

En lo referente a los elementos de innovación docente empleados en las jornadas del curso de verano, estos son numerosos y variados. Entre ellos destacan: el espacio empleado (la calle) y el contexto (urbano-ciudadanía); los recursos didácticos (póster, maquetas, esquemas, estudios de caso, etc.) y el discurso, con retroalimentación inmediata entre el docente y el discente; y el diseño o guía del trabajo del alumnado. Todos son aspectos que se complementan y apuestan por una mayor autonomía y adopción de un papel activo en el proceso de enseñanza-aprendizaje. Quizás uno de los elementos cohesionadores más novedosos es el proponer la calle como lugar didáctico y contextualizador de la experiencia docente. El alumnado era consciente así de la importancia que tenía focalizar el análisis propuesto dentro de la experiencia urbana que significaba ser ciudadano y alumno, empleando los códigos urbanos (Smardon, 2004).

A continuación, se propone profundizar en los aspectos más relevantes de las innovaciones docentes utilizadas y su relación, adaptación y aplicabilidad desde el punto de vista de los estudios de Economía y Empresa.

En la fase del seminario de transmisión de conocimientos se apostó por el uso de un espacio exterior urbano para dar una mayor visibilidad a la iniciativa y divulgar los conocimientos con un discurso cercano y accesible a la ciudadanía. El hecho de acercar el aula a la calle tiene influencia tanto en el alumnado, que está experimentando el hecho docente, como en los 
ciudadanos, que son potenciales oyentes y receptores de la misma información ofrecida al alumnado. Se utilizó como recurso didáctico el póster, ya que permite presentar resultados concretos a través de un material ilustrativo de tal forma que es posible abarcar todo el contenido con una mirada y concentrarse en los aspectos de mayor interés. Su utilización para crear debate entre los participantes y conseguir una retroalimentación es más efectiva que las exposiciones orales tradicionales (Sánchez Rodríguez et al., 2006), fomenta el aprendizaje colaborativo, en grupos pequeños y medianos (Sánchez, 2010) además de facilitar la comprensión del contenido (Guardiola, 2010). En casos como el de Empty Coruña, en los que el alumnado procede de diversas ramas de estudios, facilitar la participación, el debate y el intercambio de impresiones ayuda a adquirir unos conocimientos básicos de forma más directa, amena y cercana. La calidad didáctica de los ponentes y su estrategia por situar en el contexto urbano -donde están experimentando las clases- permiten potenciar la experiencia de enseñanza aprendizaje de los conceptos (Smardon, 2004).

Las presentaciones a través de carteles son una actividad satisfactoria y popular que los académicos continúan practicando. Sin embargo, su efectividad como forma de difusión y discusión de una investigación, más allá del momento en el que se expone, es dudosa, en el sentido de que la accesibilidad al contenido del póster de una conferencia, que no está digitalizado e indexado, puede ser complicada (Guardiola, 2010; Warren, 2018). La efectividad de un póster dependerá no sólo de sus contenidos, sino también de las necesidades personales y objetivas de sus usuarios, de tal forma que, en el caso de su utilización con fines docentes, la difusión de conocimiento a través del póster suele ser valorada positivamente por el alumnado (Sáez López \& Ruiz Ruiz, 2012).

En la fase del taller se apostó por el uso del aula en un centro cívico y se diseñó en base a un enfoque transdisciplinar de estudio de casos prácticos por equipos. Este enfoque permitió abordar los casos de estudio desde diferentes perspectivas (arquitectura, derecho, economía, etc.) aprovechando que el alumnado también presentaba perfiles profesionales y académicos diversos. Desde la perspectiva económica, y una vez impartido el seminario, se aplicó el 
modelo de aula invertida o flipped classroom a la fase del taller, una metodología que se ha utilizado anteriormente con resultados positivos en el área de estudios de economía y empresa (Barreiro-Gen, 2018; Rosa-García, 2018). El objetivo era facilitar la implicación del alumnado y del resto de agentes en un proceso de enseñanza-aprendizaje práctico y retroalimentado. Se permite así al alumnado autogestionarse y adoptar un papel activo en su formación. Los docentes actuaron como asesores a los que el alumnado trasladaba sus dudas de carácter económico-financiero. A través del uso de recursos didácticos como la elaboración de maquetas de la zona urbana asignada a cada grupo y la identificación de los actores y grupos de interés, iban reflexionando sobre la forma en la que dichos actores pueden influir en su caso de estudio. La autogestión y el aula invertida permitieron que el alumnado aumentase su implicación en el proyecto, su disposición al trabajo en equipo y su capacidad de decisión, lo que supone una mayor satisfacción con el aprovechamiento del curso y la consolidación del aprendizaje (Angelini \& García-Carbonell, 2015).

Este tipo de innovaciones podría aplicarse en las materias de economía y empresa de la UDC. El aula invertida ya se ha implementado en esta área con buenos resultados, especialmente, en aquellas materias que aplican el estudio de caso (Barreiro-Gen, 2018; Rosa-García, 2018) y que, por lo tanto, permiten una mayor autonomía del alumnado y una retroalimentación entre éste y el docente. La impartición de clases en el exterior es menos factible, aunque sí se podría trasladar la clase al mundo empresarial 0 al de instituciones económicas, con visitas que incluyan conferencias y debates.

\section{CONCLUSIONES}

El curso Empty Coruña ha generado una profunda reflexión en los docentes de la Facultad de Economía y Empresa que han participado en el mismo, sobre los esquemas tradicionales empleados para impartir las clases.

Desde el punto de vista conceptual, el curso de verano innova a partir del enfoque que propone, de carácter transdisciplinar de una problemática local (elementos urbanos vacíos en 
A Coruña), bajo un análisis intensivo que considera lo global y en espacios docentes significativos para esta problemática.

Desde el punto de vista metodológico, destaca tanto el cambio del contexto, basado en reemplazar las paredes del aula por espacios urbanos, como el enfoque activo y participativo que persigue una mayor implicación del alumnado en un proceso transdisciplinar de enseñanza-aprendizaje. Así el acercar la universidad a la ciudad, permitir el propio entorno urbano ayude directamente a cuestionarse el modelo económico que ha definido el progreso de la ciudad y ceder al alumno de dosis de responsabilidad sobre su propio aprendizaje son aspectos a tener en cuenta por los docentes para el futuro.

Como dificultad a la hora de contrastar los resultados se encontró que, al ser Empty Coruña un curso de verano con un límite máximo de 30 alumnos matriculados, el tamaño muestral no es suficiente para realizar un estudio estadístico y confirmar las valoraciones del alumnado y la efectividad de las innovaciones aplicadas. Además, es recomendable contar con un grupo de control que sirva para contrastar los resultados obtenidos, es decir, en un grupo se aplicaría una metodología tradicional y en otro las innovaciones comentadas. En base a esto, una futura línea de investigación podría ser aplicar este tipo de innovaciones adaptándolas a alguna materia que se imparte en la Facultad de Economía y Empresa de la UDC. A nivel cuantitativo, se podrían comparar los resultados de una encuesta de satisfacción en un curso anterior a aplicar estas innovaciones y en otro posterior.

No resulta fácil generalizar una experiencia innovadora, transdisciplinar y heterodoxa, como Empty Coruña, en formato de curso de verano, a otro tipo de experiencia. Aun así, es posible extraer algunas enseñanzas sobre su aplicabilidad.

- En primer lugar, las Facultades de Economía organizan frecuentemente cursos de verano, casi siempre bajo un esquema convencional de ponencias y resolución de dudas 0 cuestiones al respecto. Ejemplos como el de Empty Coruña hacen reflexionar sobre el pontencial dinamismo y diversidad metodológica aplicable en este formato de curso de verano. 
- En segundo lugar, los planes de estudios están concebidos como asignación de horas lectivas entre las diferentes áreas de conocimiento y materias más o menos estandarizadas. Quizás las reformas de los planes de estudio pudieran dejar margen para asignar algunos créditos a temas transdisciplinares y métodos menos convencionales y/o más participativos.

- En tercer lugar, dentro de las materias actuales de los planes de estudio, hay margen para introducir innovaciones docentes. Esto depende en gran medida tanto de la iniciativa de los docentes como de las autoridades académicas, generando espacios y momentos -cono los de estas jornadas de innovación docente- en los que reflexionar y concienciarse sobre la necesidad de la innovación pedagógica. En la actualidad se constata la ausencia de una "comisión de innovación docente" en la Facultad de Economía y Empresa, que podría ayudar en la dinamización de estas actividades. En el ámbito global de la UDC se crearon recientemente los grupos de innovación docente, que suponen una oportunidad para canalizar y promover iniciativas de este tipo, aunque su efectividad aún no ha sido contrastada.

\section{REFERENCIAS}

Angelini, M., \& García-Carbonell, A. (2015). Percepciones sobre la Integración de Modelos Pedagógicos en la Formación del Profesorado: La Simulación y Juego y El Flipped Classroom. Education in the Knowledge Society, 16(2), pp. 6-30.

Barreiro-Gen, M. (2018). Propuesta para la introducción del aula invertida en la docencia en Economía. Revista Educativa Hekademos, 24, pp. 52-59.

Sánchez Rodríguez et al. (2006). El método póster como herramienta de docencia en asignaturas de proyectos. Recuperado de http://hdl.handle.net/2117/6248 Guardiola, E. (2010). El póster científico. Quaderns de la Fundació Dr. Antoni Esteve, 20. https://www.raco.cat/index.php/QuadernsFDAE/article/viewFile/253632/340418 
Nimo, M., \& Durán A. (2017). Introducción ás metodoloxías activas. Plan de formación inicial. A Coruña: Universidade da Coruña.

Rosa-García, A. (2018). Aplicando la clase invertida en empresa, economía y turismo. Madrid: Editorial Dykinson.

Sáez López, J.M. \& Ruiz Ruiz, J.M. (2012). Metodología didáctica y tecnología educativa en el desarrollo de las competencias cognitivas: aplicación en contextos universitarios. Profesorado. Revista de currículum y formación del profesorado, 16(3), pp. 373-391.

Sánchez González, M. P. (2010). Técnicas docentes y sistemas de Evaluación en Educación Superior. Madrid: Narcea.

Smardon R. (2004) Streetwise Science: Toward a Theory of the Code of the Classroom. Mind, Culture, and Activity, 11(3), pp. 201-223.

Warren, R. (2018). A mapping review of poster presentation publications across time and academic disciplines. Evidence Based Library and Information Practice, 13(1), pp. 33-35.

\footnotetext{
i https://emptycoruna.net/information

ii La siguiente actividad de este proyecto fue la realización de unas jornadas ciudadanas sobre renovación y regeneración urbana en torno a los efectos de la crisis en la ciudad: A Coruña Vacía?, 30 de noviembre-2 de diciembre de 2018.

iii Además de Placido Lizancos, también formaban parte del equipo coordinador del curso Estefanía Calo, Scott Lloyd, Hans-Christian Rufer y Sonia Beltrán.

iv https://emptycoruna.net/Research-No-1-macro-economics
} 